\title{
Editorial
}

\section{Alpha-2 agonists and anaesthesia}

\section{B. Milne MD MSC FRCPC}

outflow leading to decreased heart rate, enhanced baroreflex sensitivity and depressed A-V node conduction. ${ }^{6}$ The efficacy of epidural or intrathecal $\alpha-2$ agonists stems from their action on $\alpha-2$ adrenergic receptors in the dorsal horn of the spinal cord. Noradrenergic innervation of the spinal cord arises from noradrenergic cell bodies in supraspinal sites such as the locus coeruleus. Release of endogenous norepinephrine activates $\alpha-2$ adrenoceptors, which inhibit firing of nociceptive driven cells. ${ }^{13}$

Studies by Flacke et al. demonstrated that clonidine premedication decreased the frequency and severity of hypertension and tachycardia in patients undergoing coronary artery bypass. ${ }^{14}$ Ghignone et al. reported similar findings in treated hypertensive patients undergoing various operative procedures. ${ }^{15}$ These investigations sparked an earlier editorial entitled "Alpine anaesthesia: can pretreatment with clonidine decrease the peaks and valleys?"16 Clonidine has since been used in many studies perioperatively in an endeavour to provide more "prairie-like" anaesthesia. Investigators have used clonidine to prevent hypertension during anaesthesia induction, ${ }^{17}$ decrease anaesthetic requirements, ${ }^{18}$ maintain haemodynamic stability ${ }^{19}$ and as an adjunct agent to produce deliberate hypotension. ${ }^{20}$ The use of $\alpha-2$ agonists in clinical anaesthesia has been the subject of several excellent recent reviews. ${ }^{6,21-22}$

Most studies report on the above advantages of clonidine, as well as earlier tracheal extubation, decreased shivering and preservation of respiratory function. However, disadvantages including hypertension with iv bolus injections, ${ }^{11}$ hypotension, ${ }^{23}$ bradycardia $^{11}$ and increased postoperative fluid requirements ${ }^{24}$ must be considered when using these agents. Clonidine has been shown not to decrease anxiety in women undergoing breast biopsy. ${ }^{25}$ After oral clonidine premedication in normotensive patients undergoing pelvic laparoscopy, intraoperative and postoperative hypotension was common and the authors caution against its use for routine premedication. ${ }^{23}$ Antihypertensive drug withdrawal, however, does not appear to be a problem with short-term use of clonidine.

In this issue Gaumann et al. investigated the effects of clonidine, $300 \mu \mathrm{g}$ preoperatively, in neurosurgical patients. ${ }^{26}$ They reported that clonidine decreased mean
From the Departments of Anaesthesia and Pharmacology and Toxicology, Queen's University, Kingston, Ontario, Canada. 
arterial pressure, heart rate, plasma cortisol and aldosterone but did not prevent the increase in mean arterial pressureand heart rate seen during induction of anaesthesia. The authors concluded that the overall inhibitory haemodynamic and endocrine effects of clonidine were of minimal clinical importance in ASA I or II patients. This study may represent one patient population in which the spectrum of action of this drug at a particular dose did not provide benefit. A more important effect may have been realised if the selection of patients included those who were hypertensive, elderly or receiving antihypertensive therapy, or if an increased dose had been used. This study also brings to light the important point that although observations may have reached statistical significance, this does not necessarily relate to clinical importance. Indeed, it would seem that perioperative $\alpha$-2 agonists may have the most value in procedures associated with wide swings in haemodynamic variables, such as major vascular and coronary artery surgery. Of interest in neurosurgery, clonidine may have considerable potential in the treatment of sympathetic hyperactivity that accompanies head injury reducing plasma catecholamines without decreasing cerebral blood flow. ${ }^{27}$

The perispinal administration of $\alpha-2$ agonists also shows great promise. These agents demonstrate analgesic properties for intraoperative use and in the treatment of postoperative and chronic pain. ${ }^{6,22}$ Clonidine and other $\alpha-2$ agonists given by this route produce selective and dose-dependent ${ }^{28}$ analgesia which is synergistic with coadministered opioids. ${ }^{29}$ Intrathecally, clonidine does not appear to alter spinal cord blood flow or cause neurotoxicity. The major advantage over intrathecal or epidural opiates is the lack of respiratory depression. However, side-effects similar to those following iv administration, such as hypotension, have been reported. More clinical studies are needed combining lower doses of perispinal clonidine and opiates to decrease their respective side-effects, explore their synergistic action further and perhaps lead to decreased tolerance.

In the future, new, potent, and highly selective $\alpha-2$ agonists and antagonists will be developed. Newer agents such as dexmedetomidine $e^{30-32}$ have greater $\alpha-2$ (vs $\alpha-1$ ) selectivity than clonidine, guanabenz or oxymetazoline. Dexmedetomidine has a shorter half-life and more rapid onset of action than clonidine and can decrease volatile anaesthetic requirements by more than $90 \%$ compared with $48 \%$ for clonidine. ${ }^{33}$ In addition, the development of newer, more selective $\alpha-2$ antagonists than yohimbine such as atipamezole, ${ }^{34}$ will do for $\alpha-2$ agonists what naloxone and flumazenil have done for opiates and benzodiazepines.

Thus, due to the advantageous effects of the $\alpha-2$ agonists on sympathetic activity, and their action at the spinal cord level, these drugs and their antagonists will likely find increasing use as premedicants, adjuncts to anaesthesia, perispinally, or in combination with opioids in selected patients for specific surgical procedures. Use in other areas such as treating the sympathetic cardiovascular response to head injury needs further study. Following the axiom "be not the first to use a new drug nor the last to discard an old one," more frequent selective use of these agents is warranted.

\section{Acknowledgement}

The author expresses thanks to Tara J. Kohinski CMS for preparation of the manuscript.

\section{Les agonistes alpha-2 et l'anesthesie}

Avec l'extension de l'utilisation des agonistes alpha-2, comme la clonidine, au-delà de la thérapie traditionnelle de I'hypertension, on pourrait percevoir ces agents comme une panacée. La clonidine, initialement développée comme un vasoconstricteur pour la décongestion nasale, possède maintenant des propriétés potentielles diverses qui s'étendent du sevrage de la cigarette et des opiacés, au traitement de l'angine, de l'insuffisance cardiaque, du traitement des bouffées de chaleur de la ménaupose, ainsi que des désordres neuro-psychiatriques variés et des états de douleur chronique. ${ }^{1-6}$ Alors que d'autres agonistes alpha-2 comme la xylazine, seule ou en combinaison avec la kétamine, ont été utilisés pour plusieurs années en anesthésie vétérinaire, ${ }^{7}$ leur utilisation en anesthésie clinique est relativement récente quoiqu'à date non fréquente. Les effets bénéfiques des agonistes alpha- 2 incluent le maintien de la stabilité cardio-vasculaire et des réflexes homéostatiques, la sédation, l'anxiolyse, l'analgésie, la diminution du MAC et la diminution des sécrétions salivaires. En plus, ils atténuent d'une façon efficace les hormones responsables de la réponse au stress avec réduction des catécholamines sériques, du cortisol, et de l'ADH. ${ }^{6}$ Ainsi leur utilisation en période préopératoire et dans le traitement de la douleur chronique est pharmacologiquement logique et pourrait nous introduire à l'ère de l'anesthésie par action sur des récepteurs spécifiques.

Le mécanisme d'action des agonistes alpha-2 est complexe. Depuis, le concept initial d'Ahlquist sur les récepteurs adrénergiques spécifiques ${ }^{8}$ et les travaux subséquents démontrant une inhibition de la libération de la 
norépinéphrine par des récepteurs présynaptiques qui définissent les récepteurs adrénergiques alpha-2, le tableau s'est embrouillé quelque peu. Ces récepteurs ont été récemment décrits tant post qu'extra-synapse. La situation est devenue encore plus perplexe avec la caractérisation moléculaire de plusieurs sous-types d'adrénorécepteurs alpha- $2^{9}$ et l'identification d'une substance endogène pouvent déplacer la clonidine. ${ }^{10}$ Alors que les injections intra-veineuses de clonidine produisent initialment de l'hypertension apparemment due à l'activation des récepteurs alpha-2 du muscle lisse artériel, ${ }^{11}$ l'hypotension prolongée tardive est dûe à l'augmentation de la stimulation des récepteurs alpha- 2 centraux du tronc cérébral dans des régions rostro ventrolatérales du bulbe rachidien. ${ }^{12} \mathrm{~L}$ 'activation de ces adrénorécepteurs alpha-2 centraux amène une inhibition de la stimulation sympathique provoquant une diminution de la fréquence cardiaque, une augmentation de la sensitivité des baroréflexes et une dépression de la conduction du noeud A-V. ${ }^{6}$ L'efficacité de l'administration épidurale ou intrathécale d'agonistes alpha-2 se fait grâce à leur action sur les récepteurs adrénergiques alpha-2 sur la corne dorsale de la moëlle épinière. L'innervation noradrénergique de la moëlle épinière vient des cellules noradrénergiques des sites supraspinaux tels que le locus coeruleus. La libération de norépinéphrine endogène active les adrénorécepteurs alpha-2 qui inhibent la stimulation des cellules nociceptives. ${ }^{13}$

Les études de Flacke et al. ont démontré que l'utilisation de la clonidine en prémédication diminue la fréquence et la sévérité de l'hypertension et de la tachycardie chez les patients devant subir des pontages aorto-coronariens. ${ }^{14}$ Ghignone et al. ont rapporté des trouvailles identiques chez des patients hypertendus traités, devant subir des procédures chirurgicales variées. ${ }^{15} \mathrm{Ces}$ investigations ont déclenché la publication dans le passé d'un Ėditorial intitulé "Anesthésie alpine : est-ce que le prétraitement avec la clonidine diminue les cimes et les vallées. ${ }^{16}$ La clonidine a depuis été utilisée dans plusieurs études en période périopératoire afin de founir une anesthésie plus calme et similaire plutôt "aux prairies ». Les investigateurs ont utilisé la clonidine afin de prévenir l'hypertension durant l'induction de l'anesthésie, ${ }^{17}$ de diminuer les quantities d'anesthésiques requises,$^{18}$ de maintenir une stabilité hémodynamique ${ }^{19}$ et comme un agent d'ajout, afin de produire l'hypotension provoquée. ${ }^{20}$ L'utilisation des agonistes alpha-2 en anesthésie clinique a été le sujet de plusieurs excellentes revues récentes. $6,21,22$

La majorité des articles rapportent des avantages à la clonidine comme l'extubation trachéale précoce, la diminution des frissons et la préservation de la fonction respiratoire. Cependant des désavantages tels que l'hy- pertension, lors des injections intraveineuses en bolus, ${ }^{11}$ l'hypotension, ${ }^{23}$ la bradycardie ${ }^{11}$ et l'augmentation des besoins postopératoires en liquide, ${ }^{24}$ doivent être considérés lorsqu'on utilise ces agents. La clonidne a été démontrée comme ne pouvant diminuer l'anxiété chez les femmes devant subir une biopsie du sein. ${ }^{25}$ Après une prémédication orale avec la clonidine chez des patients non hypertendus devant subir une laparascopie pelvienne, l'hypotension intra- et postopératoire était fréquente et les auteurs nous avisent du danger de l'utilisation de routine de la clonidine en prémédication. ${ }^{23}$ Avec l'utilisation de la clonidine à cours terme comme agent antihypertenseur, le syndrome du retrait n'apparait pas cependant un problème.

Dans ce numéro, Gaumann et al. ont investigué les effets de la clonidine $300 \mu \mathrm{g}$ en période préopératoire, chez des patients neuro-chirurgicaux. ${ }^{26}$ Ils ont rapporté que la clonidine a diminué la tension artérielle moyenne, la fréquence cardiaque, le cortisol plasmatique et l'aldostérone mais n'ont pas prévenu l'augmentation de la pression artérielle moyenne et de la fréquence cardiaque observée lors de l'induction de l'anesthésie. Les auteurs concluent que les effets inhibitoires hémodynamiques et endocriniens de la clonidine furent d'une importance clinique minime chez les patients ASA I et II. Cette étude peut représenter une population de patients chez qui le spectre d'action de cette drogue administrée à cette dose spécifique n'a pas fourni de bénéfice. Un effet plus important aurait pu être réalisé si la sélection des patients incluait des hypertendus, des patients âgés, ou des patients recevant une therapie antihypertensive ou une si plus grande dose a été utilisée. Cette étude amène aussi la réflexion que même si les données ont atteint une signification statistique, ceci n'implique pas nécessairement une importance clinique. En effet, il apparait que les agonistes alpha- 2 administrés en période périopératoire peuvent avoir plus de valeur dans des procédures qui sont associées à de grandes variations des données hémodynamiques telles que la chirugie vasculaire majeure et la chirurgie coronarienne. Il est intéressant de penser qu'en neurochirurgie, la clonidine pourrait avoir un potentiel considérable dans le traitement de l'hyperactivité sympathique qui accompagne le traumatisme crânien en réduisant les catécholamines plasmatiques sans diminuer le flot sanguin cérébral. ${ }^{27}$

L'administration périmédullaire des agonistes alpha-2 a aussi démontré de grandes promesses. Ces agents possèdent des propriétés analgésiques pour l'utilisation peropératoire et pour le traitement de la douleur chronique et postopératoire. ${ }^{6,22} \mathrm{La}$ clonidine et d'autres agonistes alpha-2 ont été administrés par cette route produisant une analgésie sélective et dépendant de la dose ${ }^{28}$ qui était synergique avec la coadministration d'opiacés. ${ }^{29}$ Il parait 
que la clonidine intrathécale n'altère pas le flot sanguin médullaire et n'est pas neurotoxique. Son avantage majeur sur les opiacés en administration épidurale ou intrathécale serait le manque de dépression respiratoire. Cependant les effets secondaires similaires à l'adminstration intraveineuse tels que l'hypotension, ont été rapportés. D'autre études cliniques sont nécessaires combinant de faibles doses de clonidine périmédullaire et d'opiacés afin de diminuer leurs effets secondaires respectifs, d'explorer l'action synergique et peut-être de diminuer la tolérance.

Dans le futur, de nouveaux agonistes alpha-2 hautement sélectifs et puissants et des antagonistes spécifiques seront développés. De nouveaux agents tels que la dexmédétomidine ${ }^{30-32}$ ont une sélectivité alpha-2 supérieure à la clonidine, la guanabenz ou l'oxymétazoline. La dexmédétomidine a une demi-vie plus courte et un début d'action plus rapide que la clonidine et peut diminuer le besoin d'un agent anesthésique volatil de plus de $90 \%$ comparativement à $48 \%$ pour la clonidine. ${ }^{33}$ En plus, le développement de nouveaux antagonistes alpha-2 plus sélectifs que la yohimbine tels que l'atipamézole ${ }^{34}$ sera pour les antagonistes alpha- 2 ce que la naloxone et la flumazénil sont pour les opiacés et les benzodiazépines.

Ainsi grâce à leurs effets avantageux sur la diminution de l'activité sympathique et leur action au niveau de la moelle épinière, les agonistes alpha-2 et leurs antagonistes trouveront probablement un usage croissant en prémédication, comme agent d'adjonction à l'anesthésie en administration périmédullaire seuls ou en combinaison avec les opiacés chez des patients sélectionnés pour des procédures chirurgicales spécifiques. Leur utilisation dans d'autres domaines tel que le traitement de la réponse sympathique cardiovasculaire, lors du traumatisme crânien demande à être investigué. Si l'on suit l'axiome « ne soit pas le premier à utiliser une nouvelle drogue ni le dernier à rejeter une vieille », une utilisation sélective plus fréquente de ces agents est de mise.

\section{References}

1 Gourlay SG, McNeil JJ. Antismoking products. Med J Aust 1990; 153: 699-707.

2 Hartmann F, Poirier MF, Bourdel MC, Loo H, Lecomte $J M$, Schwartz JC. Comparison of acetorphan with clonidine for opiate withdrawal symptoms. Am J Psychiatry 1991; 148: 627-9.

3 Thomas MG, Quiroz AL, Rice JC, Sander GE, Giles TD. Antianginal effects of clonidine. J Cordiovasc Pharmacol 1986; 8: (Suppl 3) S69-S75.

4 Giles TD, Iteld BJ, Mautner RK, Rognoni PA, Dillenkoffer $R L$. Short term effects of intravenous clonidine in congestive heart failure. Clin Pharmacol Ther 1981; 30: 724-8.

5 Nagamani $M$ Kelver ME, Smith ER. Treatment of menopausal hot flashes with transdermal administration of clonidine. Am J Obstet Gynecol 1987; 156: 561-5.

6 Maze $M$, Tranquilli $W$. Alpha-2 adrenoceptor agonists: defining the role in clinical anesthesia. Anesthesiology 1991; 74: 581-605.

7 Popilskis SJ, Oz MC, Gorman P, Florestal A, Kohn DF. Comparison of xylazine with tiletamine-zolazepam (Telazol) and xylazine-ketamine anesthesia in rabbits. Lab Anim Sci 1991; 41: 51-3.

8 Ahlquist RP. A study of the adrenotropic receptors. Am J Physiol 1948; 153: 586-600.

9 Harrison JK, Pearson WR, Lynch K. Molecular characterization of $\alpha 1$ and $\alpha 2$ adrenoceptors. Trends Pharmacol Sci $1991 ; 12: 62-7$.

10 Bousquet $P$, Feldman J, Tibirica $E$ et al. New concepts on the central regulation of blood pressure. Alpha-2 adrenoceptors and imidazoline receptors. Am J Med 1989; 87: $3 \mathrm{C}-10 \mathrm{~S}$.

11 Hoffman $B B$, Lefkowitz $R J$. Catecholamines and sympathomimetic drugs. In: Gilman AG, Rall TW, Nies AS, Tylor P (Eds.). The Pharmacological Basis of Therapeutics. New York: Pergamon Press, 1990: 187-220.

12 Hong $M$, Milne $B$, Loomis $C$, Jhamandas $K$. In vivo catechol activity in the rostral ventrolateral medulla: effects of the $\alpha-2$ adrenergic agonist dexmedetomidine. Can J Anaesth 1991; 38: A10.

13 Yaksh TL. Pharmacology of spinal adrenergic systems which modulate spinal nociceptive processing. Pharmacol Biochem Behav 1985; 22: 845-58.

14 Flacke JW, Bloor BC, Flacke WE et al. Reduced narcotic requirement by clonidine with improved hemodynamic and adrenergic stability in patients undergoing coronary bypass surgery. Anesthesiology 1987; 67: 11-9.

15 Ghignone M, Calvillo $O$, Quintin L. Anesthesia and hypertension: the effect of clonidine on perioperative hemodynamics and isoflurane requirements. Anesthesiology $1987 ; 67: 3-10$.

16 Longnecker DE. Alpine anesthesia: can pretreament with clonidine decrease the peaks and valleys. Anesthesiology $1987 ; 67: 1-2$.

17 Ghignone M, Quintin L, Duke PC, Kehler CH, Calvillo O. Effects of clonidine on narcotic requirements and hemodynamic response during induction of fentanyl anesthesia and endotracheal intubation. Anesthesiology 1986; 64: 36-42.

18 Segal IS, Jarvis DA, Duncan SR, White PF, Maze M. Clinical efficacy of oral-transdermal clonidine combinations during the perioperative period. Anesthesiology 1991; 74: $220-5$. 
19 Quintin L, Bonnet F, MacQuin I, Szekely B, Becquemin $J P$, Ghignone $M$. Aortic surgery: effect of clonidine on intraoperative catecholaminergic and circulatory stability. Acta Anaesthesiol Scand 1990; 34: 132-7.

20 Toivonen J, Kaukinen S. Clonidine premedication: a useful adjunct in producing deliberate hypotension. Acta Anaesthesiol Scand 1990; 34: 653-7.

21 Flacke JW. Opioid anesthesia and the alpha-2 agonists. American Society of Anesthesiologists Annual Refresher Course Lectures, 1990, 235.

22 Eisenach J. Alpha-2 adrenergic agonists. American Society of Anesthesiologists Annual Refresher Course Lectures, 1990, 126.

23 Wright PMC, Carabine UA, McClune S, Orr DA, Moore J. Preanesthetic medication with clonidine. $\mathrm{Br} \mathrm{J}$ Anaesth 1990; 65: 628-32.

24 Quintin L, Roudot F, Roux C et al. Effect of clonidine on the circulation and vasoactive hormones after aortic surgery. Br J Anaesth 1991; 66: 108-15.

25 Pouttu J, Scheinin B, Rosenberg PH, Viinamiki $O$, Scheinin $M$. Oral premedication with clonidine: effects on stress responses during general anaesthesia. Acta Anaesthesiol Scand 1987; 31: 730-4.

26 Gaumann DM, Tassony E, Rivest RW, Fathi $M$, Reverdin $A F$. Cardiovascular and endocrinological effects of clonidine premedication in neurosurgical patients. Can J Anaesth 1991; This issue.

27 Payen D, Quintin L, Plaisance P, Chiron B, Lhoste F. Head injury: clonidine decreases plasma catecholamines. Critical Care Medicine 1990; 18: 392-5.

28 Milne B, Cervenko FW, Jhamandas KH, Sutak $M$. Intrathecal clonidine: anesthesia and effect on opiate withdrawal in the rat. Anesthesiology 1985; 62: 34-8.

29 Drasner K, Fields $H L$. Synergy between the antinociceptive effects of intrathecal clonidine and systemic morphine in the rat. Pain 1988; 32: 309-12.

30 Doze VA, Chen B-X, Maze $M$. Dexmedetomidine produces a hypnotic-anesthetic action in rats via activation of central alpha-2 adrenoceptors. Anesthesiology 1989; 71: 75-9.

31 Stenberg $D$. Physiological role of $\alpha 2$-adrenoceptors in the regulation of vigilance and pain: effect of medetomidine. Acta Vet Scand 1989; 85: 21-8.

32 Kallio A, Scheinin $M$, Koulu $M$ et al. Effects of dexmedetomidine, a selective alpha 2-adrenoceptor agonist, on hemodynamic control mechanisms. Clin Pharmacol Ther 1989; 46: 33-42.
33 Segal IS, Vickery RG, Walton JK, Doze VA, Maze M. Dexmedetomidine diminishes halothane anesthetic requirements in rats through a postsynaptic alpha- 2 adrenergic receptor. Anesthesiology 1988; 69: 818-23.

34 Virtanen $R$. Pharmacological profiles of medetomidine and its antagonist, atipamezole. Acta Vet Scand 1989; 85: 29-37. 